\title{
Iterated Nearest Neighbors and Finding Minimal Polytopes*
}

\author{
David Eppstein ${ }^{1}$ and Jeff Erickson ${ }^{2}$ \\ ${ }^{1}$ Department of Information and Computer Science, University of California, \\ Irvine, CA 92717, USA \\ eppstein@wormwood.ics.uci.edu \\ ${ }^{2}$ Computer Science Division, University of California, \\ Berkeley, CA 94720, USA \\ jeffe@cs.berkeley.edu
}

\begin{abstract}
We introduce a new method for finding several types of optimal $k$-point sets, minimizing perimeter, diameter, circumradius, and related measures, by testing sets of the $O(k)$ nearest neighbors to each point. We argue that this is better in a number of ways than previous algorithms, which were based on high-order Voronoi diagrams. Our technique allows us for the first time to maintain minimal sets efficiently as new points are inserted, to generalize our algorithms to higher dimensions, to find minimal convex $k$-vertex polygons and polytopes, and to improve many previous results. We achieve many of our results via a new algorithm for finding rectilinear nearest neighbors in the plane in time $O(n \log n+k n)$. We also demonstrate a related technique for finding minimum area $k$-point sets in the plane, based on testing sets of nearest vertical neighbors to each line segment determined by a pair of points. A generalization of this technique also allows us to find minimum volume and boundary measure sets in arbitrary dimensions.
\end{abstract}

\section{Introduction}

A number of recent papers have discussed problems of selecting, from a set of $n$ points, the $k$ points optimizing some particular criterion [2], [14], [20]. Criteria that have been studied include diameter [2], variance [2], area of the convex hull [20], convex hull perimeter [14], [20], and rectilinear diameter and perimeter [2].

\footnotetext{
* Portions of this paper were presented at the 3rd and 4th ACM-SIAM Symposia on Discrete Algorithms [17], [19]. This paper includes work done while Jeff Erickson was at the University of California at Irvine. David Eppstein's research was partially supported by NSF Grant CCR-9258335.
} 
Such problems are useful in clustering, line detection, statistical data analysis, and other geometric applications.

We study and improve known algorithms for these problems. We also introduce dynamic versions of these problems, in which the optimum set must be maintained as new points are inserted. Our methods further generalize to higher-dimensional versions of these problems. Our techniques apply to all of the problems cited above.

Many of these problems were previously solved using the following method, originally developed by Dobkin et al. [14]. An ad hoc algorithm was determined, with time bounded by a polynomial $O\left(n^{c}\right)$. Then it was shown that the optimum $k$-point set is contained in the set of points labeling a single region of the order- $O(k)$ Voronoi diagram. Constructing the Voronoi diagram and searching the $O(k n)$ such regions takes a total time of $O\left(n \log n+k^{c+1} n\right)$. Aggarwal et al. [2] reduced the number of regions to be searched from $O(k n)$ to $O(n)$. Thus the time becomes $O\left(n \log n+k^{c} n\right)$. However, an anomaly remains in these time bounds: if $k$ is $\Theta(n)$, the time is worse than the original $O\left(n^{c}\right)$ by a factor of $n$. Thus at some point the device of higher-order Voronoi diagrams becomes worthless, and one must use a simpler algorithm.

We argue that, in this formulation, Voronoi diagrams should be replaced by sets of the $O(k)$ nearest neighbors to each point. There are several reasons why we believe this. First, the reduction to $O(n)$ regions to be searched is immediate, and avoids the complicated analysis of Aggarwal et al. [2].

Second, by finding neighbors of neighbors, we show that the number of regions can be further reduced to $O(n / k)$, improving the time bounds by a factor of $k$ and eliminating the anomaly described above.

Third, our time bounds can be improved in a different way. The $k$ nearest neighbors can be found in time $O(k n \log n)$, using Vaidya's algorithm [34]. For the rectilinear $\left(L_{1}\right.$ or $\left.L_{\infty}\right)$ metric, we further improve this to $O(n \log n+k n)$ on a random access machine. Thus we get faster time bounds in the plane, even for problems such as circumradius for which the reduction to Voronoi diagrams is immediate.

Fourth, our method lends itself well to dynamization. As points are inserted one at a time, the neighbors of each new point may be computed quickly using standard techniques. In contrast, the Voronoi diagram may change by as many as $\Omega(n)$ edges at each insertion. Dynamic algorithms have been studied for many important geometric optimization problems, such as the closest pair, diameter, minimum spanning tree, and convex hull, but this is the first time that dynamic algorithms have been described for minimum measure subset problems.

Fifth, our approach generalizes to higher dimensions in a way that does not work for Voronoi diagrams. In dimension $d$ even first-order Voronoi diagrams can have complexity $\Omega\left(n^{[d / 2\rceil}\right)$; whereas, the nearest negibhors can still be found in time $O(n \log n)$ using Vaidya's algorithm [34].

Finally, by applying an old combinatorial result of Erdös and Szekeres [21], we can generalize our techniques to find minimum measure convex polygons and polytopes.

A variant of our technique also yields new algorithms for finding minimum area $k$-point sets in the plane, or minimum boundary measure or volume $k$-point sets in arbitrary dimensions. Our boundary measure algorithm is a natural 
generalization of our minimum perimeter result. Instead of using the neighbors to each point, we let each set of $r$ points in the set define a particular polytope, for some constant $r$ defined by the measure we are trying to minimize, and we examine the nearest neighbors to each polytope thus defined. In particular, we show that the minimum area $k$-point set is contained in the $O(k)$ nearest vertical neighbors to a line segment determined by two of its points.

\section{New Results}

We present algorithms for the following problems:

- We find the $k$ nearest rectilinear $\left(L_{1}\right.$ or $\left.L_{\infty}\right)$ neighbors to each of a set of $n$ points in the plane, in time $O(n \log n+k n)$, improving the previous $O(k n \log n)$ bound [34].

- We find the $k$ nearest vertical neighbors to each of the $O\left(n^{2}\right)$ line segments determined by pairs of points in an $n$-point set, in total time $O\left(k n^{2}+n^{2} \log n\right)$

- Given a set of $n$ points in the plane, we find the $k$-point set minimizing perimeter, $L_{\infty}$ perimeter, circumradius, diameter, $L_{\infty}$ diameter, variance, or area. Our results are summarized in the first column of Table 1 . We improve all previous results [2], [14], [20], except for variance and area, which we improve for certain values of $k$.

- We maintain minimal point sets in the plane as points are inserted, under a variety of "one-dimensional" measures. Our results are summarized in the second column of Table 1 . No previous bounds are known for any of these problems.

- Given a set of $n$ points in $\mathbb{R}^{d}$, with $d>2$, we find the $k$-point set minimizing circumradius, diameter, $L_{\infty}$ diameter, variance, boundary measure, $L_{\infty}$ boundary measure, or volume. Our results are summarized in Table 2 . We improve previous algorithms for circumradius and variance based on Voronoi diagrams, which run in time $O\left(n^{d+1}\right)$ [2]. No previous bounds were known for the other problems.

Table 1. New results for finding minimum measure $k$-point sets, given $n$ points in the plane. ( $\varepsilon$ is an arbitrarily small positive constant.)

\begin{tabular}{lll}
\hline \multicolumn{1}{c}{ Measure } & \multicolumn{1}{c}{ Static time bound } & Dynamic time bound \\
\hline Perimeter & $O\left(n \log n+k^{3} n\right)$ & $O\left(k^{4}+\log ^{2} n\right)$ \\
$L_{\infty}$ perimeter & $O\left(n \log n+k^{2} n\right)$ & $O\left(k^{3}+\log ^{2} n\right)$ \\
Circumradius & $O(n \log n+k n \log k)$ & $O\left(k^{2} \log k+\log ^{2} n\right)$ \\
Diameter & $O\left(n \log n+k^{2} n \log ^{2} k\right)$ & $O\left(k^{3} \log ^{2} k+\log ^{2} n\right)$ \\
$L_{\infty}$ diameter & $O\left(\min \left\{n \log n+k n, n \log ^{2} n\right\}\right)$ & $O\left(k \log ^{2} k+\log ^{2} n\right)$ \\
Variance & $O\left(k^{3 / 2} n \log n+k^{3 / 2+\varepsilon} n\right)$ & $O\left(k^{3+\varepsilon}+\log ^{2} n\right)$ \\
\hline Area & $O\left(n^{2} \log n+k^{3} n^{2}\right)$ & \\
\hline
\end{tabular}


Table 2. New results for finding minimum measure $k$-point sets, given $n$ points in $\mathbb{R}^{d}$, for all $d>2$.

\begin{tabular}{ll}
\hline \multicolumn{1}{c}{ Measure } & \multicolumn{1}{c}{ Time bounds } \\
\hline Circumradius & $O\left(k n \log n+k^{d-1} n \log ^{2} k\right)$ \\
Diameter & $O\left(k n \log n+2^{O(k)} n\right)$ \\
$L_{\infty}$ diameter & $O\left(k n \log n+k^{d / 2-1} n \log ^{2} k\right)$ \\
Variance & $O\left(k^{(d+1) / 2} n \log n+k^{O\left(d^{2}\right)} n \log k\right)$ \\
\hline Boundary measure & $O\left(n^{d}+2^{O(k)} n^{d-1}\right)$ \\
$L_{\infty}$ boundary measure & $O\left(n^{d}+k^{2 d-1} n^{d-1}\right)$ \\
Volume & $O\left(k n^{d} \log ^{d+1} n+2^{O(k)} n^{d}\right)$ \\
\hline
\end{tabular}

- We generalize all of our results to $k$-point convex polygons and polytopes. We derive time bounds with the same dependence on $n$ as the corresponding $k$-point set algorithms, but with an exponential dependence on $k$. We know of no previous results for these problems, except for an $O\left(k n^{3}\right)$ time bound on finding minimum area or perimeter $k$-gons [20], which we improve for small $k$.

\section{Rectilinear Nearest Neighbors}

We begin by describing a data structure for finding $m$ rectilinear nearest neighbors in the plane. In the $L_{1}$ metric, above and to the right of any point $p$, points $(x, y)$ are sorted by distance to $p$ by the values of the function $x+y$. If we sort all points by these values, the nearest neighbors above and to the right of each point will be a subsequence of this sorted list. We combine neighbors from each of the four directions to find the nearest neighbors overall.

Our data structure is in the form of a balanced binary tree over the points, sorted by their $y$-coordinates. The tree root covers all $n$ points, and for each tree node with $i$ points we split the points into two slabs, consisting of the top $i / 2$ and the bottom $i / 2$ points. We build a data structure for each slab, and recursively subdivide slabs until we reach sets of a single point. Each input point will be in $O(\log n)$ slabs, and the points above and to the right of any query point $p$ can be interpreted as the union of points to the right of $p$ in $O(\log n)$ slabs above $p$.

We assume $m$ is fixed. Without loss of generality $m>\log n$. (Otherwise, we build our data structure to find $\log n$ nearest neighbors, from which we can quickly choose the nearest $m$.) In each slab we wish to determine, for a query point $p$, the $m$ nearest points to the right of $p$. If we did this for all slabs, we would generate $O(m \log n)$ neighbors, and queries would be slower than we wish. Instead, we partition the neighbors into chunks of $\Theta(m / \log n)$ points. Our data structure enables us to find each succeeding chunk quickly, and we then combine chunks from different slabs to give the final set of $m$ neighbors.

Within a single slab, we sweep from left to right, maintaining a list of points 
ordered by $x+y$. As we sweep across each point in the slab, we add it to the list. The positions to add new points into the list can be found in time $O(n)$ if the points are already sorted by $x$-coordinate. We would like our data structure to reconstruct the state of this list at each time in the sweep. This is a persistent offline data structure problem [18], in which we perform a number of updates (insertions into a linked list) and must then query different versions of the data structure (the list at different times in the sweep).

We maintain, at each point in the left to right sweep, a partition of the sorted list of points into chunks of between $m / \log n$ and $2 m / \log n$ points each. When a new point is inserted in the list, it is added to a chunk. When this addition causes a chunk to have too many points, it is split into smaller chunks. As we only need remember at most $m$ neighbors to each query point, we only need keep $\log n$ chunks, so as one chunk is split another chunk may be removed from the end of the list.

To remember these manipulations we store the list of points in each chunk just before the chunk is split, and the list of all $\log n$ chunks at the same time. To find the neighbors for a query point $p$, we determine the next time $t$ after our left-to-right sweep crosses $p$, at which some chunk is split. We then step through the sequence of chunks existing at time $t$. Each chunk contains between $m / \log n$ and $2 m / \log n$ points, of which at least $m / \log n$ existed at the last time the chunk was split and hence are to the right of $p$. We eliminate the other points to the left of $p$. Thus in time $O(m / \log n)$ we can find each successive set of $\Omega(m / \log n)$ neighbors in the slab.

The time and storage for remembering the points in each chunk is $O(n)$. However, if $m$ is small there are $O(n)$ times at which a chunk may split, and hence $O(n \log n)$ storage for remembering the sequence of chunks at each time. We remove this unwanted logarithmic factor with a data structure for maintaining lists of $O(\log n)$ elements in a persistent offline manner.

Lemma 3.1. Given a sequence of $n$ insert and delete operations on a list, such that the list length is always $O(\log n)$, we can construct in time and space $O(n)$ a data structure such that, for any version of the list, we can step through the list in time O(1) per step.

Proof. Break the sequence into $O(n / \log n)$ subsequences of $O(\log n)$ operations each, and treat each subsequence separately. Within a subsequence, there are $O(\log n)$ items initially, and $O(\log n)$ items inserted. List all items by processing all the insert operations and none of the delete operations. Represent this list as an array of pointers to items, so that the item in a given position can be found in $O(1)$ time. Now represent each version of the list by an $O(\log n)$-bit integer, in which a one bit represents the presence of the item at that position. Each insert or delete can be performed with $O(1)$ steps of integer arithmetic on a random access machine, as can the operation of moving from one element to the next in a given version of the list.

By analogy to the atomic heaps of Fredman and Willard [23] we call this data 
structure an atomic list. This completes the description of each slab, which we summarize below.

Lemma 3.2. Given $n$ points in the plane, sorted from left to right, we can in time and space $O(n)$ construct a data structure for which, given a value $x$, we can find the points with the smallest values of $x+y$, in chunks of $O(m / \log n)$ points at a time, in time $O(m / \log n)$ per chunk.

To finish the description of our data structure, we combine results from the $O(\log n)$ slabs into which space above the query is divided. We use a priority queue of one chunk from each slab. Each chunk's priority is the largest value of $x+y$ for any point in the chunk. We remove chunks one by one from the queue; when we remove a chunk we insert the next chunk from the same slab. Once we have removed chunks totaling at least $m$ points, any remaining neighbors will have smaller values of $x+y$ than the priorities of the chunks left in the queue. Such points must be in chunks already in the queue, and we remove these chunks as well. This gives us $O(\log n)$ chunks and hence $O(m)$ potential neighbors. We reduce this to $m$ neighbors using a linear-time selection algorithm. Using a global list of all points, sorted by $x+y$, we can represent priorities as $O(\log n)$-bit integers, so we can perform priority queue operations in $O(1)$ time using atomic heaps [23].

Lemma 3.3. For any fixed $m$, we can preprocess a set of $n$ points in the plane, in time and space $O(n \log n)$, so that the $m$ nearest rectilinear neighbors to any query point can be found in time $O(m+\log n)$.

Proof. The query time is $O(m+\log n)$, once we have determined the version of the chunk list to use in each slab. For each slab we maintain an index from the left to right order of points into this sequence of versions. We also index, for each slab, the relation between positions in the left to right order of points in the slab, and the same positions in the two smaller slabs into which it is divided. The position of the query point in the root slab can be found by binary search, after which we can follow the indices to find the list versions for all $O(\log n)$ slabs queried in $O(\log n)$ time. The time to construct each slab is $O(n)$ assuming the points are sorted from left to right. This sorted order can be maintained as slabs are split recursively, in $O(n \log n)$ total time. Each slab requires space $O(n)$. Thus all slabs can be constructed in time and space $O(n \log n)$.

Theorem 3.1. We can find the $m$ nearest rectilinear neighbors to each of a set of $n$ points in the plane, in time $O(n \log n+m n)$ and space $O(n \log n)$.

Remark. Our nearest neighbor algorithm relies on the constant-time manipulation of $O(n \log n)$-but integers, in both the atomic lists and atomic heaps. Thus, our time bound holds only in the RAM model of computation. In the algebraic decision tree model our time bound climbs by a factor of $O(\log \log n)$. 


\section{Iterated Nearest Neighbors}

We now show that in any point set, in any dimensions, there is some point for which there are few neighbors of neighbors. We state the result more generally, in terms of spheres satisfying certain properties. Given two spheres $A$ and $B$, we say that $A$ is entirely within $B$ if the closure of $A$ is contained in the interior of $B$.

Lemma 4.1. Let $\mathscr{S}$ be a set of spheres, so that no sphere is entirely within another sphere, and so that no sphere contains more than $m$ centers of spheres. Let $S$ be the smallest sphere in $\mathscr{S}$, and let $U$ be the union of spheres in $\mathscr{S}$ having centers in $S$. Then $U$ contains $O(m)$ sphere centers.

Proof. Let $R$ denote the radius of $S$. Because no sphere in $U$ contains $S$, it follows that $U$ is contained in a sphere of radius $3 R$. This larger sphere can be partitioned into $O(1)$ regions, each with diameter $R$. If any of these regions contained more than $m$ centers, any sphere centered in such a region either would contain too many centers or would be smaller than $S$.

This result applies more generally to any family of homothetic convex bodies, and hence to "spheres" in any metric. We apply this result to sets of $m$ nearest neighbors as follows. Given a point set, put a sphere around each point at a radius determined by its $m$ th nearest neighbor. This sphere will contain exactly the $m$ nearest neighbors of the point, and the set of all such spheres will satisfy the conditions of the lemma. Therefore there is some point for which the $m$ nearest neighbors have $O(m)$ neighbors altogether.

This suggests the following algorithm outline. Suppose we can prove that the optimal $k$-point set (according to some specified criterion), if it contains a point, is contained in the $m$ nearest neighbors of that point. Sort the points by the size of their neighbor spheres. Collect the neighbors of the points in the smallest neighbor sphere, search for the optimal set among them, and throw out the $m+1$ points in the sphere. Repeat the preceding step until all points are gone, but if a smallest neighbor sphere ever contains fewer than $k$ points, we throw out its center immediately, since that point cannot possibly be in the optimal set.

Lemma 4.2. Let $\mu$ be a measure having the property that the minimum measure $k$-point set is contained in the $m$ rectilinear nearest neighbors of each of its points, and let $T(m)($ resp. $S(m))$ be the time (resp. space) required to find the optimal k-point set among $m$ points. Then, given a set of $n$ points in $\mathbb{R}^{d}$, we can find the $k$-point subset minimizing $\mu$, in time $O(m n \log n+n T(m) / k)$ and space $O(m n+S(m))$, or in time $O(n \log n+m n+n T(m) / k)$ and space $O(n \log n+m n+S(m))$ if $d=2$.

Proof. By our results above, the rectilinear nearest neighbors can be found in time $O(n \log n+m n)$ and space $O(n \log n)$ in the plane. In higher dimensions we use Vaidya's algorithm [34]. We only call the ad hoc algorithm when the neighbor sphere contains $k$ or more points, and those points are immediately discarded. Thus, the ad hoc algorithm is called at most $\lceil n / k\rceil$ times, each time on a set of 
size $O(m)$ by the previous lemma. These two operations dominate the time and space bounds.

We claim that at any point in the algorithm, if the minimal set has not yet been found, it contains only points that have not been thrown out. We prove this claim by induction; it is clearly true when the algorithm starts. If any point $p$ is in the set, then by assumption, the set is contained in $p$ 's neighbor sphere. If this sphere contains fewer than $k$ points, $p$ cannot possibly be in the set, and we can safely discard it. The only other time $p$ could be thrown out is immediately after we search for the minimal set among $p$ 's remaining neighbors. If the minimal set contains $p$, then by the inductive hypothesis, this search will find it.

The same algorithm outline still works if the minimum measure set is contained in the nearest neighbors of each of its points, under any fixed metric. Thus, in higher dimensions, we could use Euclidean neighbors instead of rectilinear neighbors without changing the time bounds.

\section{Minimizing One-Dimensional Measures}

\subsection{Perimeter}

We first demonstrate our technique on the minimum perimeter $k$-point set problem. The problem is to find, given a set of $n$ points in the plane, a set of $k$ points for which the perimeter of the convex hull is minimized. This was previously solved in $O\left(k^{2} n \log n+k^{5} n\right)$ time by Dobkin et al. [14]; this can be improved to $O\left(n \log n+k^{4} n\right)$ using the techniques of Aggarwal et al. [2]. Both algorithms use a dynamic programming algorithm, requiring time $O\left(\mathrm{kn}^{3}\right)$ and space $O(k n)$, as a subroutine within each region of a certain high-order Voronoi diagram [14]. We use this algorithm as a subroutine within each set of nearest neighbors.

Lemma 5.1. If a point $p$ is in the minimum perimeter $k$-point set, then the set is contained in the $O(k)$ nearest rectilinear neighbors of $p$.

Proof. Let $q$ be the farthest point from $p$ in the optimal set. Then the entire set fits in a circle around $p$, of radius $|p q|$, and the perimeter must be at least $2|p q|$. However, we can cover the circle with 16 squares of perimeter $|p q|$; if $q$ is not among the $16 k$ rectilinear nearest neighbors of $p$, then some square must contain at least $k$ points, and would supply a $k$-point set with smaller perimeter.

We point out that this lemma is also true for Euclidean neighbors, or neighbors under any fixed metric, with only a change in the constant factor. This is true for almost all of our nearest-neighbor results.

Theorem 5.1. We can find the minimum perimeter $k$-point subset of a set of $n$ points in the plane, in time $O\left(n \log n+k^{3} n\right)$ and space $O\left(n \log n+k n+k^{2}\right)$. 
Proof. This is a direct application of the two-dimensional case of Lemma 4.2. We have $m=O(k)$ by the previous lemma, and $T(m)=O\left(\mathrm{~km}^{3}\right)$ and $S(m)=O(\mathrm{~km})$ from [14].

This algorithm generalizes to minimize perimeter in any metric, but we can do better in $L_{\infty}$. The minimum $L_{\infty}$ perimeter $k$-point set is the set enclosable in the minimum perimeter axis-aligned rectangle. Aggarwal et al. [2] solve this problem in time $O\left(k^{2} n \log n\right)$. We use their $O\left(n^{3}\right)$-time brute-force algorithm as a subroutine.

Theorem 5.2. We can find the minimum $L_{\infty}$ perimeter $k$-point subset of a set of $n$ points in the plane, in time $O\left(n \log n+k^{2} n\right)$ and space $O(n \log n+k n)$.

\subsection{Circumradius}

We now describe our algorithms for finding the $k$-point set contained in the smallest sphere, given a set of $n$ points in $\mathbb{R}^{d}$. We improve previous time bounds, due to Aggarwal et al. [2], of $O\left(n \log n+k^{2} n\right)$ in the plane and $O\left(n^{d+1}\right)$ in higher dimensions. Their algorithms are based on higher-order Voronoi diagrams.

First we develop a new algorithm to use as a subroutine within each neighbor set. Consider the related problem of placing a fixed-size sphere so that it covers the maximum number of a given set of points. Once we have a solution to this problem, we can apply Megiddo's parametric search technique [29] to find the smallest sphere whose optimal placement covers $k$ (or more) points.

Lemma 5.2. We can find the minimum circumradius $k$-point subset of $a$ set of $n$ points in $\mathbb{R}^{d}$, in time $O\left(n^{d} \log ^{2} n\right)$ and space $O\left(n^{d} \log n\right)$, or in time and space $O\left(n^{2} \log n\right)$ if $d=2$.

Proof. First consider the sphere placement problem. We fix each set of $d-1$ points and rotate a sphere around its affine hull, stopping whenever a point enters or leaves the sphere. Each sweep requires time $O(n \log n)$. Degenerate cases, where the optimal sphere cannot be forced to be tangent to $d$ points, are handled in total time $O\left(n^{d-1}\right)$. Thus, the entire sweep algorithm takes time $O\left(n^{d} \log n\right)$. In the plane we can solve this problem in time and space $O\left(n^{2}\right)$, using a more complicated algorithm developed by Chazelle and Lee [8].

To find minimum circumradius sets, we apply parametric searching with Cole's weighted median strategy [10]. Our sweep algorithm can be parallelized to run in $O(\log n)$ steps on $O\left(n^{d}\right)$ processors. Thus, the total time is $O\left(n^{d} \log ^{2} n\right)$ in general, and $O\left(n^{2} \log n\right)$ in the plane. The parametric search technique requires the construction of an AKS sorting network [3] with $O\left(n^{d}\right)$ inputs, one for each processor used by the parallel implementation of the fixed-parameter algorithm. This network can be built in time and space $O\left(n^{d} \log n\right)[3]$. 
Lemma 5.3. If a point $p$ is in the minimum circumradius $k$-point set, then the set is contained in the $O(k)$ rectilinear nearest neighbors of $p$.

Proof. Let $R$ be the optimal circumradius. The minimum circumradius set is contained in an axis-aligned hypercube of width $4 R$, centered at $P$. This hypercube can be partitioned into $(2 \sqrt{d})^{d}=O(1)$ axis-parallel hypercubes with circumradius $R$, none of which can contain more than $k$ points.

Theorem 5.3. We can find the minimum circumradius $k$-point subset of a set of $n$ points in $\mathbb{R}^{d}$, in time $O\left(k n \log n+k^{d-1} n \log ^{2} k\right)$ and space $O\left(k n+k^{d} \log k\right)$, or in time $O(n \log n+k n \log k)$ and space $O\left(n \log n+k n+k^{2} \log k\right)$ if $d=2$.

\subsection{Diameter}

The diameter of a set is the largest distance between any two points in the set. In the plane Aggarwal et al. [2] show how to find the minimum diameter $k$-point set in time $O\left(n \log n+k^{2.5} n \log k\right)$. Unfortunately, we know of no fully polynomial algorithm for this problem in higher dimensions, so we are forced to use a brute-force approach.

Lemma 5.4. If a point $p$ is in the minimum diameter $k$-point set, then the set is contained in the $O(k)$ rectilinear nearest neighbors of $p$.

Proof. Let $D$ be the optimal diameter. The minimum diameter set is contained in an axis-parallel hypercube of width $2 D$, centered at $p$. This hypercube can be partitioned into a constant number of smaller hypercubes with diameter $D$, none of which can contain more than $k$ points.

Theorem 5.4. We can find the minimum diameter $k$-point subset of a set of $n$ points in $\mathbb{R}^{d}$, in time $O\left(k n \log n+2^{O(k)} n\right)$ and space $O(k n)$, for all $d>2$.

We can do considerably better than this in the plane. Aggarwal et al. [2] solve this problem by reducing it to one of finding a maximum independent set in a certain bipartite graph. For bipartite graphs, the maximum independent set and maximum matching are closely related (their cardinalities add to the size of the point set) so matching techniques can be applied to this problem.

We improve on the algorithm of Aggarwal et al. by solving a dynamic matching problem. Given a point set $S$, and a distance $D$, let the graph $G_{D}(S)$ be defined as follows. The vertices of $G_{D}(S)$ are simply the points in $S$. An edge $(p, q)$ exists in the graph exactly when $|p q|>D$; that is, the graph connects points that are sufficiently far apart.

Lemma 5.5. Given a set $S$ of $n$ points, such that $G_{D}(S)$ is bipartite, and $a$ maximum matching in $G_{D}(S)$, we can insert or delete a single point in $S$ and update 
the maximum matching, in time $O(n \log n)$ and space $O(n)$, provided $G_{D}(S)$ remains bipartite.

Proof. The update can only change the matching cardinality by one. If the update is a deletion of a matched point, we remove its edge from the matching and mark its mate as unmatched. Then whether the update is an insertion or a deletion, the remaining problem is to find a single alternating path connecting two unmatched points. If such a path is found, the matching size can be increased by changing the unmatched edges in it to matched edges, and vice versa.

We go through a process of marking points as odd or even. A point is labeled odd (even) if it can be reached from an unmatched vertex by an alternating path of odd (even) length. In each case we remember the last edge on the path, so that the entire path can be reconstructed quickly. Once we have performed this labeling, the existence of an alternating path can be tested by testing if any two even points share an edge. This can be done in $O(n \log n)$ time by finding the farthest pair of even points.

We maintain a data structure for a point set $P$ with the following operations:

(1) Given a point $p$, find some point in $P$ farther than $D$ from $p$, or report that no such point exists.

(2) Delete a given point from $P$.

As noted by Aggarwal et al. [2], these operations can be performed in $O(\log n)$ amortized time and linear space using the circular hull data structure of Hershberger and Suri [25].

We start the labeling process by marking each unmatched point as even (it has a zero length path to an unmatched point). We build the data structure above, letting $P$ consist of all unmarked points (initially, that is simply the matched points). We then process each marked point in turn, maintaining a queue of points that require processing. Processing an odd point consists simply of marking its match even, adding it to the queue, and removing it from $P$. We process the even points as follows. While an unmarked point adjacent to the even point exists, we mark it odd, add it to the queue, and remove it from $P$. Such a point can be found using the find operation described above.

Once the queue is empty, all points are either marked or unreachable via an alternating path. The number of data structure operations is $O(n)$, as each find operation either discovers a new point to be marked and removed from $P$, or it is the last such operation performed in processing a given point. Therefore the total time used is $O(n \log n)$. The circular hull structure and the queue both use linear space, so the overall space bound is linear.

Lemma 5.6. We can find the minimum diameter $k$-point subset of a set of $n$ points in the plane, in time $O\left(n^{3} \log ^{2} n\right)$ and space $O(n)$.

Proof. Each point can be the endpoint of $O(n)$ possible diameters; we select among them using binary search. To test a given diameter $D$ associated with a 
point $p$, we want to know whether there is some $k$-point set with diameter $|p q|$ shorter than $D$. If so, the set is contained in the lune formed by intersecting two circles of diameter $D$, one centered on $p$ and one centered at distance $D$ from $p$. We sweep a lune around $p$, covering in turn $O(n)$ different point sets; we must test if any of these sets contains a small diameter $k$-point subset.

As noted by Aggarwal et al. [2], if $S$ is the point set contained in a given lune, then $G_{D}(S)$ is bipartite, and a subset of $S$ with diameter less than $D$ is exactly an independent set in $G_{D}(S)$. If $M$ is the maximum matching in $G_{D}(S)$, the size of the maximum independent set is $|S|-|M|$. Thus to test if there is a large subset with small diameter, we may compute this matching. We do this for all $O(n)$ positions of the lune around $p$, in time $O\left(n^{2} \log n\right)$, using the dynamic matching algorithm of Lemma 5.5.

For each point we perform a binary search among its $O(n)$ associated diameters, and there are $O(n)$ points for which this search must be performed. Thus, the total time used is $O\left(n^{3} \log ^{2} n\right)$.

Combining this algorithm with Lemmas 4.2 and 5.4, we have the following result.

Theorem 5.5. We can find the minimum diameter $k$-point subset of a set of $n$ points in the plane, in time $O\left(n \log n+k^{2} n \log ^{2} k\right)$ and space $O(n \log n+k n)$.

\section{4. $L_{\infty}$ Diameter}

The algorithms in the previous two sections generalize to circumradius and diameter in any metric, but we can make a significant improvement in $L_{\infty}$. The minimum $L_{\infty}$ diameter (equivalently, minimum $L_{\infty}$ circumradius) $k$-point set is the set enclosable in the smallest axis-aligned hypercube. In the plane Aggarwal et al. [2] give an algorithm for this problem, based on higher-order $L_{\infty}$ Voronoi diagrams, that takes time $O\left(k^{2} n \log n\right)$.

Our approach is almost identical to that used to find minimum circumradius sets. We start with the problem of placing a fixed-size axis-aligned hypercube so that it covers the maximum number of points. Once we solve this problem, we can parametrize it to find the smallest axis-parallel hypercube that covers at least $k$ points. Instead of parametric search, we use a much simpler binary search among the possible $L_{\infty}$ diameters.

Lemma 5.7. We can find the minimum $L_{\infty}$ diameter $k$-point subset of a set of $n$ points in $\mathbb{R}^{d}$, in time $O\left(n^{d / 2} \log ^{2} n\right)$ and space $O\left(n^{d / 2}\right)$.

Proof. Finding the optimal placement of a hypercube is equivalent to finding the deepest point in an arrangement of hypercubes. We can easily adapt an algorithm of Overmars and Yap [32], originally applied to Klee's measure problem, to find the deepest point in an arrangement of axis-aligned boxes in time $O\left(n^{\mathrm{d} / 2} \log n\right)$ and space $O\left(n^{d / 2}\right)$. 
To find the optimal hypercube size, we search along each coordinate axis as follows. We sort the points by the appropriate coordinate, and define a triangular matrix $M$ of coordinate differences. These differences are potential $L_{\infty}$ diameters. We do not actually build $M$, since that would require time $\Omega\left(n^{2}\right)$, but we can access any entry in constant time. We binary search through $M$ for the optimal diameter. Since the rows and columns of $M$ are sorted, we can select any element in time $O(n \log n)$ [22]. Thus, each step of the search is dominated by Overmars and Yap's algorithm, and the entire search requires time $O\left(n^{d / 2} \log ^{2} n\right)$.

Theorem 5.6. We can find the minimum $L_{\infty}$ diameter $k$-point subset of a set of $n$ points in $\mathbb{R}^{d}$, in time $O\left(k n \log n+k^{d / 2-1} n \log ^{2} k\right)$ and space $O\left(k n+k^{d / 2}\right)$, or in time and space $O(n \log n+k n)$ if $d=2$.

Our planar algorithm's time and space bounds are dominated by the rectilinear nearest-neighbors subroutine. For $k=\Omega\left(\log ^{2} n\right)$, the " ad hoc" algorithm of Lemma 5.7 is both faster and more space-efficient.

\subsection{Variance}

The variance of a set of points is defined as the sum of the squares of the distances between pairs of points, divided by the number of points in the set. Equivalently, the variance is the sum of the squares of the distances from each point to the centroid of the set [2].

Lemma 5.8. If a point $p$ is in the minimum variance $k$-point set, then the set is contained in the $O\left(k^{d / 2+1}\right)$ rectilinear nearest neighbors of $p$.

Proof. Let $V$ and $R$ be the variance and circumradius of the minimum variance set, and let $p$ be any point in the set. We easily verify that $2 R^{2}<V \leq k R^{2}$. The set is contained in an axis-parallel hypercube centered at $p$ with width $4 R$. We can partition this hypercube into $O\left(k^{d / 2}\right)$ hypercubes of width $2 R \sqrt{2 / d k}$, and thus of circumradius $R \sqrt{2 / k}$. If any of these spheres contain $k$ points, their variance is at most $2 R^{2}$, which is less than $V$.

Aggarwal et al. [2] prove that the minimum variance $k$-point set corresponds to one of the cells in the $k$ th-order Voronoi diagram of the original $n$ points and derive an algorithm that uses time $O\left(n \log n+k^{2} n\right)$ in the plane. Agarwal and Matoušek [1] recently discovered an algorithm for constructing planar order- $k$ Voronoi diagrams in time $O\left(k n^{1+\varepsilon}\right) .{ }^{1}$ Combining their algorithm with our techniques, we can find minimum variance sets in the plane in time $O\left(n \log n+k^{2+\varepsilon} n\right)$, which is slightly worse than the existing bound.

\footnotetext{
${ }^{1}$ Throughout this paper $\varepsilon$ represents an arbitrarily small positive constant. Multiplicative constants hidden by the $O$-notation may depend on $\varepsilon$.
} 
Lemma 5.9. Let $p$ be a point in the minimum variance $k$-point set, and let $V$ be the set's variance. Suppose, for some constant $c>0$, the distance between $p$ and the set's centroid is $c \sqrt{V / k}$. Then the set is contained in the $O\left(c k^{(\mathrm{d}+1) / 2}\right)$ nearest Euclidean neighbors of $p$.

Proof. Let $S$ be the sphere, centered at the optimal set's centroid, which just contains the set, and let $R$ be the radius of $S$. $S$ contains exactly $k$ points [2]. Then $S$ is contained in a sphere centered at $p$ with radius $R+2 c \sqrt{V / k}$. The space between the two spheres can be covered by $O\left(c k^{(d-1) / 2}\right)$ spheres of radius $\sqrt{V / k}$, none of which can contain $k$ points.

The two previous bounds are tight in the worst case. Consider a sphere $S_{1}$ of radius $\sqrt{k}$, containing a smaller sphere $S_{2}$ of radius $\sqrt{k} / 2$ tangent to $S_{1}$. There is a cluster of $k-2$ points with arbitrarily small variance around the center of $S_{2}$, but excluding the center itself. The surface of $S_{2}$ and the space between the two spheres are both filled with as many clusters of $k / 2$ points as possible, such that every two clusters have at least unit distance between them. One of these clusters contains the center of $S_{1}$; another contains the tangent point of the two spheres. The minimum variance set consists of the large cluster, the center of $S_{1}$, and the tangent point. For each point $p$ in this set, every sphere centered at $p$ that contains the set also contains $\Omega\left(k^{(d+1) / 2}\right)$ other points, and the set contains the $\Theta\left(k^{d / 2+1}\right)$ th neighbor of the center of $S_{1}$.

To find the minimum variance set quickly, we need to find a center point within radius $c \sqrt{V / k}$ of the optimal set's centroid, for some constant $c>0$, so that we can search for the optimal set among its $O\left(c k^{(d+1) / 2}\right)$ nearest neighbors. We describe an algorithm the finds a set of $O(n / k)$ points, at least one of which is a center point.

Theorem 5.7. We can find the minimum variance $k$-point subset of a set of $n$ points in the plane, in time $O\left(k^{3 / 2} n \log n+k^{3 / 2+\varepsilon} n\right)$ and space $O\left(k n+k^{5 / 2}\right)$.

Proof. We begin by finding the $k / 2$ nearest Euclidean neighbors to every point, in time $O(k n \log n)$ and space $O(k n)$. We repeatedly find the point $p$ with the smallest neighbor sphere. If neither $p$ nor any of the neighbors of $p$ are already marked noncentral, we make $p$ as a potential center point and mark its neighbors as noncentral. Each central point marks $k / 2$ noncentral points, so this process gives us $O(n / k)$ potential center points. The entire marking process requires time $O(n \log n+k n)$.

Most of the points in the minimal set are within $\sqrt{2 V / k}$ of the centroid, so every point within this radius has at least $k / 2$ neighbors within $2 \sqrt{2 V / k}$. Let $p$ be one of these points. When the marking algorithm reaches $p$, one of two things happens: 
(1) We could mark $p$ as a potential center point.

(2) We could ignore $p$ because $p$ or one of its neighbors is marked noncentral, in which case some point within $5 \sqrt{2 V / k}$ of the centroid is already marked central.

Thus, at least one of the potential center points is an actual center point.

After we find the potential center points, we find the $O\left(k^{3 / 2}\right)$ nearest Euclidean neighbors of each potential center point in time $O\left(k^{3 / 2} n \log n\right)$ and space $O(n)$. We then test each of the $O(n / k)$ neighbor sets in time $O\left(k^{5 / 2+\varepsilon}\right)$ and space $O\left(k^{5 / 2}\right)$ using Agarwal and Matoušek's Voronoi diagram algorithm [1].

This algorithm matches or improves previous time bounds for all $k$ in $O\left(n^{\varepsilon}\right) \cap \Omega\left(\log ^{2} n\right)$. For smaller values of $k$, the $O\left(n \log n+k^{2} n\right)$-time algorithm of Aggarwal et al. is faster. For larger values of $k$, Agarwal and Matoušek's Voronoi diagram algorithm is faster. Finally, for $k=\Omega\left(n^{1-\varepsilon}\right)$, the fastest algorithm is based on another Voronoi algorithm of Chazelle and Edelsbrunner [6] and runs in time $O\left(n^{2} \log ^{2} n\right)$.

Mulmuley describes an algorithm that constructs the $k$ th-order Voronoi diagram of a set of $n$ points in $\mathbb{R}^{d}$, in time $O\left(k^{\lceil(d+1) / 2} n^{\lfloor(d+1) / 2 \downarrow} \log n+k^{d} n^{2}\right)$ [30]. To find minimum variance sets in higher dimensions, we use Mulmuley's algorithm as a subroutine within each neighbor set. We improve the previous time bound of $O\left(n^{d+1}\right)[2]$.

Theorem 5.8. We can find the minimum variance $k$-point subset of a set of $n$ points in $\mathbb{R}^{d}$, in time $O\left(k^{(d+1) / 2} n \log n+k^{v(d)-1} n \log k\right)$ and space $O\left(n+k^{v(d)}\right)$, where $v(d)=\left(d^{2}+3 d+4\right) / 4$ if $d$ is even, and $\left(d^{2}+4 d+3\right) / 4$ if $d$ is odd.

\section{Dynamization}

We now show how to turn our planar algorithms into dynamic data structures, that can maintain the minimum measure $k$-point set as points are inserted. Our algorithm is simply to maintain a data structure that can determine, for each new point, its $O(k)$ nearest neighbors. Then if that point is part of a set improving the previous optimum, that set will be a subset of these neighbors, and can be found using the methods already described.

Lemma 6.1. Let $\mu$ be a measure having the property that the minimum measure $k$-point set is contained in the $m$ nearest neighbors of each of its points, and let $T(m)($ resp. $S(m))$ be the time (resp. space) required to find the optimal k-point set among $m$ points. Then, in the plane we can maintain the $k$-point set minimizing $\mu$ as points are inserted in time $O\left(T(m)+\log ^{2} n+m \log n\right)$ per insertion and space $O(n \log n+S(m))$. 
Proof. We apply a standard dynamic-to-static reduction technique for decomposable searching problems [4] to the rectilinear nearest neighbor data structure of Lemma 3.3.

Theorem 6.1. We can maintain the minimum measure $k$-point set in the plane as points are inserted, with the following insertion times: $O\left(k^{4}+\log ^{2} n\right)$ for perimeter, $O\left(k^{3}+\log ^{2} n\right)$ for $L_{\infty}$ perimeter, $O\left(k^{2} \log k+\log ^{2} n\right)$ for circumradius, $O\left(k^{3} \log ^{2} k+\log ^{2} n\right)$ for diameter, $O\left(k \log ^{2} k+\log ^{2} n\right)$ for $L_{\infty}$ diameter, and $O\left(k^{3+\varepsilon}+\log ^{2} n\right)$ for variance.

We can dynamize our higher-dimensional results in a similar manner, using a dynamic nearest-neighbor data structure of Agarwal and Matoušek [1], with results that are just slightly better than brute force.

\section{Nearest Vertical Neighbors}

We now consider the problem nearest vertical neighbors for points and line segments; we use this as a subroutine in our minimum area algorithm.

Given a point $p$ and a nonvertical line $l$, the vertical distance $d(p, l)$ is simply the length of a vertical line segment connecting $p$ and $l$. The nearest vertical neighbor to $l$ from a point set $P$ is the point $p \in P$ minimizing the vertical distance to $l$.

The connection between this concept and minimum area polygons is as follows. If a triangle is formed by connecting point $p$ to the endpoints of a line segment $s$, where $s$ is contained in line $l$, the area of the triangle is $c \cdot d(p, l)$, where $c$ is half the length of the projection of $s$ onto the $x$-axis. Therefore the point in $P$ forming the minimum area triangle with $s$ is the nearest vertical neighbor of $l$. This observation was used to develop $O\left(n^{2}\right)$ algorithms for the minimum area triangle problem [7], [16], [15].

We can tighten this characterization as follows. Let $\triangle p q r$ be the minimum area triangle, and assume that the vertical projection of $r$ is between those of $p$ and $q$. Then, as before, $r$ is the nearest neighbor of line $p q$, but the vertical segment connecting $r$ and line $p q$ actually touches segment $p q$. In other words, $r$ is within the slab defined by vertical lines through $p$ and $q$. In general we say $p$ is a neighbor of segment $s$ if it appears vertically above or below $s$, as in this case $r$ appears above or below segment $p q$. Then the triangle problem can be solved by finding, for each segment $p q$, the nearest neighbors above and below $p q$. Computing nearest neighbors to segments is harder than the original problem of nearest neighbors to lines but it generalizes to minimum area $k$-gons in a way that does not work for nearest neighbors to lines.

Given a set of $n$ points and a segment $s$ (which may not be determined by two of the points) we say that the $k$ nearest vertical neighbors above (below) $s$ are those $k$ points above (below) the line through $s$, and within the slab defined by vertical lines through the endpoints of $s$, such that no other point with the same restrictions is closer to $s$. We compute these $k$ nearest neighbors as follows. 


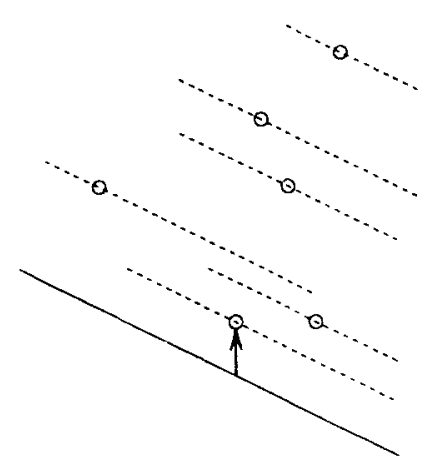

(a)

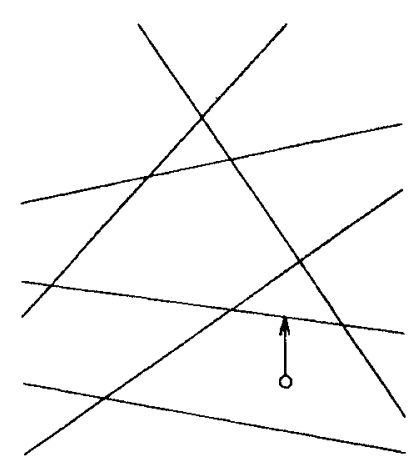

(b)

Fig. 1. Finding nearest neighbors between lines and points: (a) primal, nearest point neighbor to a line; (b) dual, vertical ray shooting in a line arrangement.

First, suppose we only care about the restriction that the points be above or below the line $l$ through $s$; this is the earlier problem of nearest neighbors to a line. By geometric duality, we can transform the line to a point and the point set to a line arrangement; Fig. 1(a) and (b) illustrates this transformation. Point-line vertical distances are the same in the dual as they were in the primal. Therefore the dual nearest neighbor is the first line encountered by a vertical ray sent from the dual point $l$. The $k$ nearest neighbors are the first $k$ lines encountered. Thus we can find these neighbors by ray shooting in the dual arrangement.

This does not quite solve the problem we posed, because we have not dealt with the requirement that points be above or below the segment $s$, only that they be above or below the line containing $s$. To solve this we sort the points by their horizontal projections. As well as building an arrangement and ray-shooting data structure for the entire point set, we also build structures for the first and second halves of the sorted lists of points, and so on recursively. Each data structure corresponds to a vertical slab of the points; for each such structure there are two smaller structures corresponding to slabs containing half as many points.

Then, for each query segment $s$, we can find a set of $O(\log n)$ slabs that contain exactly those points above and below $s$. We can solve the $k$ nearest vertical neighbors problem by performing ray-shooting queries within each slab, and selecting the best $k$ points found.

We now describe the data structures which allow us to perform these rayshooting operations and therefore find the nearest vertical neighbors. Rather than build a data structure that allows line segments to be tested in arbitrary order, we test the segments in left-to-right order of the points dual to the lines containing them. This allows us to perform our algorithm as a plane sweep of the dual-line arrangement; that is, we sweep a vertical line from left to right across the dual plane, and perform line segment neighbor finding queries and data structure updates as the vertical line crosses appropriate features in the arrangement.

At any point in the algorithm, the sweep line will cross all of the $n$ dual lines, 
Our data structure for a single slab (corresponding to a single dual-line arrangement) simply consists of an array of $n$ elements, listing those lines in the vertical order of their points of intersection with the sweep line. Then a vertical rayshooting query along the sweep line could be performed by a binary search in the array, to locate the starting point among the dual lines. Successive queries would then take $O(1)$ time by simply moving up to the next element in the array. The order of the dual lines changes exactly when the sweep line crosses an intersection between two dual lines. The change consists simply of swapping two adjacent elements in the array. The rays at which we wish to shoot correspond to segments $p q$, which are also found as the intersection of two lines dual to $p$ and $q$. Each successive intersection can be found in $O(\log n)$ time, by keeping a priority queue of the $n-1$ possible intersections between adjacent elements in the array.

Now let us consider putting several slabs together again. Starting each rayshooting query by binary searching in each slab separately would take $O\left(\log ^{2} n\right)$ time per segment. We can reduce this time, by using another data structure to relate locations in different slabs to each other. Recall that for each slab, corresponding to an arrangement of some $m$ dual lines, there are two smaller slabs with $m / 2$ dual lines each. The sweep line in the large slab is divided into $m+1$ regions by the $m$ lines crossing it. Each region in the large slab corresponds to part of a region in each of the smaller slabs. We keep another array, of $m+1$ elements, listing the correspondence between regions of the large and small slabs. This correspondence only changes when an intersection occurs in the large slab; other intersections will rearrange the correspondence of lines to regions but will not change the numbering of the regions. For each intersection of two lines, we only need to update the correspondence for the region between the lines. Thus again each update takes $O(1)$ time per slab.

Now we can use these arrays to locate the point dual to each segment in the $O(\log n)$ slabs we wish to search. The point is an intersection in the outer slab containing all $n$ points, and its location will already be known when the sweep line crosses that intersection. Then, while we have a location within a slab that contains points not above or below the queried segment, we need to move to locations in the two child slabs. This can be done simply by looking in the appropriate arrays, in constant time per move. In this way it takes $O(\log n)$ time to find the initial locations for ray shooting in each of the $O(\log n)$ appropriate slabs.

Once we have found the initial locations for vertical ray shooting, we can find each successive vertical neighbor of the segment simply by moving from element to adjacent element in the appropriate array. However, we must somehow combine the neighbors found in different slabs. To do this, we use a final array, which tells us for each line the position of that line as it crosses the sweep line in the root slab of all $n$ points. This array is updated as before by swapping two elements per intersection encountered. Using the array, we can compare neighbors from different slabs, by examining their positions in the sweep line. Each vertical neighbor for the line segment must be found by selecting among $O(\log n)$ candidates, one from each slab, each of which can be thought of as an integer having $O(\log n)$ bits, representing the position in the sweep line. 
This section can be performed in constant time per operation, using atomic heaps [23].

Theorem 7.1. Given $n$ points, we can enumerate all point sets found as the $k$ nearest vertical neighbors of each segment formed by a pair of points, in total time $O\left(n^{2} \log n+k n^{2}\right)$ and space $O(n \log n)$.

Proof. Each intersection of two dual lines, causing a search from the corresponding segment as well as updates to the data structures, can be selected in time $O(\log n)$ from a priority queue of possible intersections. Each search for $k$ nearest neighbors takes time $O(\log n)$ to find the initial positions for the ray shooting, and $O(1)$ time per neighbor found. Each update takes constant time per slab, and involves changes in $O(\log n)$ slabs (only those slabs containing both dual lines that intersect to cause the update). Therefore the total time for searches and updates in $O\left(n^{2}(\log n+k)\right)$. Each dual line is involved in $O(\log n)$ slabs, and uses constant space per slab, so the total space is $O(n \log n)$.

Remark. Again, since our algorithm uses atomic heaps, our time bound holds only in the RAM model of computation. In the algebraic decision tree model the time bound increases by a factor of $O(\log \log n)$.

\section{Minimizing Area}

We now describe how to use the data structure of the previous section to find $k$-point sets with minimum area convex hulls. The problem was previously solved by Eppstein et al. [20] in time $O\left(k n^{3}\right)$ and space $O\left(k n^{2}\right)$.

Lemma 8.1. If $p_{\mathrm{L}}$ and $p_{\mathrm{R}}$ are the leftmost and rightmost points of the minimum area $k$-point set, respectively, then every other point in the set is one of the $2 k-4$ nearest neighbors above or below the segment $p_{\mathrm{L}} p_{\mathrm{R}}$.

Proof. Let $q$ be the point farthest from $p_{\mathrm{L}} p_{\mathrm{R}}$ in the optimal set. Then the area of the set is at least that of $\triangle p_{\mathrm{L}} q p_{\mathrm{R}}$. Consider the parallelogram with two vertical sides through $p_{\mathrm{L}}$ and $p_{\mathrm{R}}$, one side equal to $p_{\mathrm{L}} p_{\mathrm{R}}$, and the remaining side parallel to $p_{L} p_{R}$ and through $q$. We can divide this parallelogram vertically into two smaller parallelograms, each with area equal to that of $\triangle p_{\mathrm{L}} q p_{\mathrm{R}}$. If either parallelogram contains $k$ points, they would supply a $k$-point set with smaller area. Thus, the large parallelogram contains at most $2 k-2$ points, including $p_{\mathrm{L}}$ and $p_{\mathrm{R}}$.

For example, Fig. 2(a) shows a set of five points, with $\triangle p_{L} q p_{R}$ outlined. Figure 2(b) shows $7=2 \cdot 5-3$ points above $p_{L} p_{R}$, five of which are in one small parallelogram.

This immediately gives us our algorithm for finding the minimum area $k$-point 


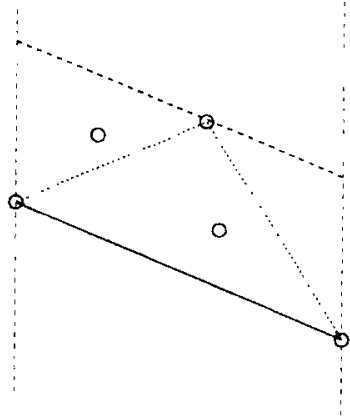

(a)

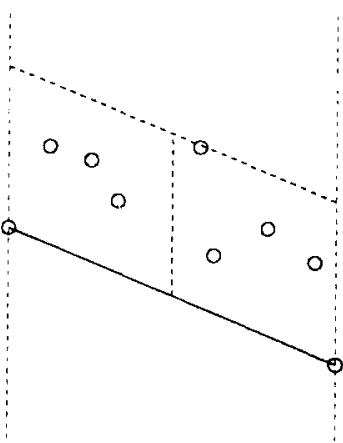

(b)

Fig. 2. Nearest neighbors to $p_{\mathrm{L}} p_{\mathrm{R}}$ : (a) five-point set with triangle to furthest point; (b) seven nearest neighbors have a five-point subset in one parallelogram.

set. We simply examine each possible segment $p_{\mathrm{L}} p_{\mathrm{R}}$, and find the $2 k-4$ neighbors above and below it. That gives us a set of $4 k-6$ points, in which we can find the minimum $k$-point set in time $O\left(k^{4}\right)$ using the algorithm of Eppstein et al. [20]. Thus we achieve a total time of $O\left(n^{2} \log n+k^{4} n^{2}\right)$.

The $O\left(k^{4}\right)$ term in this bound can be improved. First note that the convex hull of the minimum $k$-point set is the union of the convex hulls above and below $p_{\mathrm{L}} p_{\mathrm{R}}$, and the area of the convex hull is the sum of the areas above and below $p_{\mathrm{L}} p_{\mathrm{R}}$. Each of these two hulls must be a minimum $j$-point set, among those sets containing both $p_{\mathrm{L}}$ and $p_{\mathrm{R}}$, for $j$ equal to the number of points in the respective sets. So if we can compute all minimum such $j$-point sets, for $j<k$, we can combine the possible top and bottom sets in a further step taking time $O(k)$. In fact the algorithm of Eppstein et al. computes all these sets. Further, it works by trying all possible choices of the bottommost point in the set; for each such choice the algorithm takes $O\left(k^{3}\right)$ time. However, in our situation we know the bottommost point: it is either $p_{\mathrm{L}}$ or $p_{\mathrm{R}}$. Therefore we can compute the optimum $k$-point set among the neighbors of segment $p_{\mathrm{L}} p_{\mathrm{R}}$ in time $O\left(k^{3}\right)$.

Theorem 8.1. We can find the minimum area $k$-point subset of a set of $n$ points in the plane, in time $O\left(n^{2} \log n+k^{3} n^{2}\right)$ and space $O\left(n \log n+k^{3}\right)$.

This improves the previous time bound of $O\left(k n^{3}\right)[20]$ whenever $k=O(\sqrt{n})$. Our space bound is always an improvement over the previous $O\left(\mathrm{kn}^{2}\right)$ [20].

\section{Minimizing Volume and Boundary Measure}

In this section we demonstrate a natural generalization of our minimum area algorithm to arbitrary dimensions. We also generalize our results for finding minimum perimeter sets in the plane. 
Let $T$ be some $r$-dimensional polytope in $\mathbb{R}^{d}$, with $r<d$. Given a point $p$, we define the orthogonal distance from $p$ to $T$ to be the Euclidean distance from $p$ to its orthogonal projection onto aff( $(T)$, which we denote $p^{\prime}$. We call $p$ an orthogonal neighbor of $T$ if and only if $p^{\prime} \in T$. We can compute the nearest orthogonal neighbors to any polytope with fixed complexity in linear time.

Given a set of points $A$ in $\mathbb{R}^{d}$, and an arbitrary point $p_{0} \in A$, we define the series of extremal points, extremal simplices, and bounding boxes of $A$ with respect to $p_{0}$, denoted $p_{i}, S_{i}$, and $B_{i}$, respectively. While these sequences depend on the initial point $p_{0}$, the properties we derive hold for all initial points.

We define $B_{0}=S_{0}=p_{0}$. For each $1 \leq i \leq d, p_{i}$ is the point in $A$ farthest from the affine hull of $S_{i-1} . S_{i}=\operatorname{conv}\left(S_{i-1}, p_{i}\right) . B_{i}$ is the convex hull of two copies of $B_{i-1}$, one containing $p_{i}$ and one an equal distance from $B_{i-1}$ in the opposite direction, situated so that $B_{i-1} \subset B_{i}$, and adjacent facets of $B_{i}$ meet at right angles. For any set $A$, we have $S_{d}(A) \subset \operatorname{conv}(A) \subset B_{d}(A)$. See Fig. 3 for an example.

Volume and boundary measure share the following property. For some constant $r$, the minimum measure $k$-point set is contained in the $m$ nearest orthogonal neighbors to the bounding box of its first $r$ extremal points (with respect to any point in the set). For measures with this property, we have the following algorithm outline for finding minimum measure sets. For each set of $r$ points, there are $r$ possible bounding boxes. For each box, we find its $m$ nearest orthogonal neighbors, and search for the minimum measure set among them.

Lemma 9.1. Let $\mu$ be a measure having the property that the minimum measure $k$-point set $A$ is contained in the $m$ nearest orthogonal neighbors of $B_{r}(A)$, and let $f(m)$ be the time required to find the optimal k-point set among $m$ points. Then, given $a$ set of $n$ points in $\mathbb{R}^{d}$, we can find the $k$-point subset minimizing $\mu$, in time $O\left(n^{r+1}+n^{r} f(m)\right)$.

We know of no fully polynomial-time algorithm to find minimum volume or boundary measure sets, except in the plane [2], [14], [20]. A naive algorithm runs in time $O\left(\left(\begin{array}{l}n \\ k\end{array}\right) k^{\lfloor d / 2\rfloor}\right)$, by explicitly computing the convex hull of every $k$-point subset [5]. We use this algorithm as a subroutine.

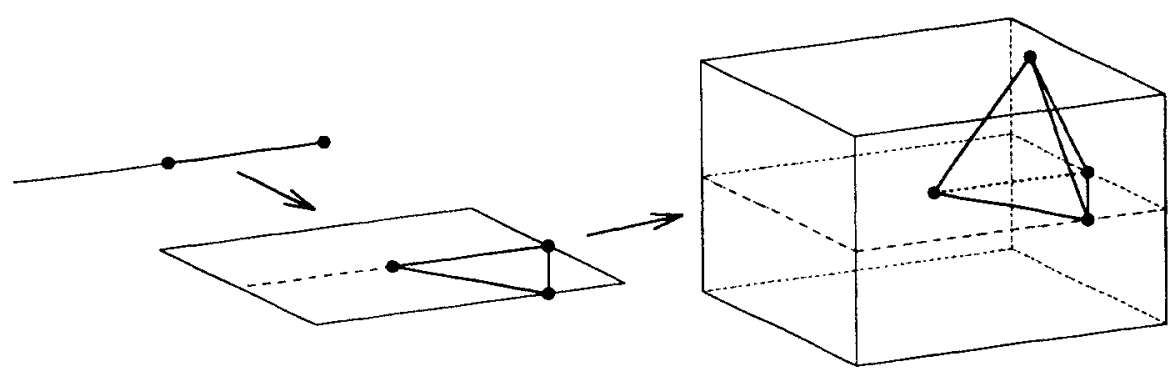

Fig. 3. Extremal points, extremal simplices, and bounding boxes in $\mathbb{R}^{3}$. 
Throughout this section we let $|A|$ and $|\partial A|$ denote the volume and boundary measure of the convex hull of $A$. The following lemma relates the volumes of bounding boxes and extremal simplices.

Lemma 9.2. For all $A \subset \mathbb{R}^{d},\left|B_{d}(A)\right|=2^{d} d !\left|S_{d}(A)\right|$.

Proof. The volume of a $d$-dimensional cone is $b h / d$, where $b$ is the $(d-1)$ dimensional measure of the base and $h$ is the distance between the apex and the affine hull of the base. The volume of a $d$-dimensional box with the same base measure and height is $b h$.

We prove the lemma by induction. The lemma holds (trivially) when $d=0$. Let $h_{d}$ denote the distance between $p_{d}$ and aff $\left(S_{d-1}\right)$. Using the volume formulae above, we have $\left|S_{d}\right|=h_{d}\left|S_{d-1}\right| / d$ and $\left|B_{d}\right|=2 h_{d}\left|B_{d-1}\right|$. Therefore, $\left|B_{d}\right| /\left|S_{d}\right|=$ $2 d\left|B_{d-1}\right| /\left|S_{d-1}\right|$. The closed form follows directly from the inductive hypothesis.

\subsection{Boundary Measure}

Given a set $A$ in $\mathbb{R}^{d}$, we define its bounding cylinder $C(A)$ as the set of points no farther orthogonally from $B_{d-2}(A)$ than $p_{d-1}(A)$, and we define $B^{\prime}(A)$ as the smallest box containing $C(A)$. We have $S_{d-1}(A) \subset \operatorname{conv}(A) \subset C(A) \subset B^{\prime}(A)$. See Fig. 4 .

The following lemma relates the boundary measure of any set $A$ with the boundary measure of $B^{\prime}(A)$.

Lemma 9.3. For all $A \subset \mathbb{R}^{d},\left|\partial B^{\prime}(A)\right|<2^{d-1} d !|\partial A|$.

Proof. $\quad B^{\prime}$ has $2 d$ facets. Four of the facets have measure equal to $\left|B_{d-1}\right|$. Let $h_{i}$ denote the distance between $p_{i}$ and $\operatorname{aff}\left(S_{i-1}\right)$. Since $h_{d-1}<h_{i}$ for all $i<d-1$, the

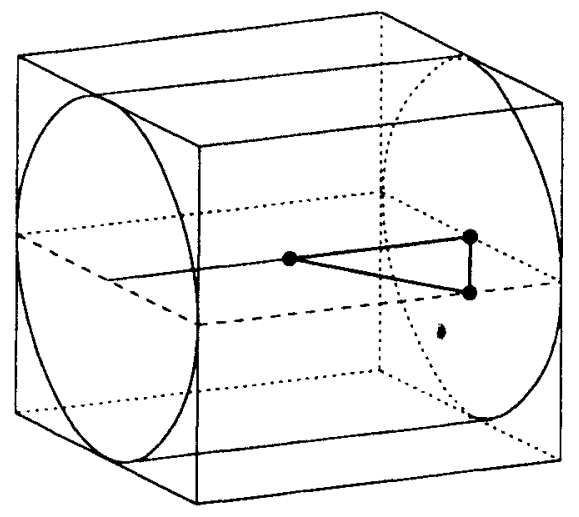

Fig. 4. A bounding cylinder and its box in $\mathbb{R}^{3}$. 
rest of the facets of $B^{\prime}$ have measure smaller than $\left|B_{d-1}\right|$. Therefore, $\left|\partial B^{\prime}\right|<$ $2 d\left|B_{d-1}\right|=2^{d} d\left|S_{d-1}\right|$, by Lemma 9.2. The lemma follows from the observation that $|\partial A|>2\left|S_{d-1}(A)\right|$.

Lemma 9.4. The minimum boundary measure set $A$ is contained in the $O(k)$ nearest orthogonal neighbors of $B_{d-2}(A)$.

Proof. Let $s(d)$ denote $\left\lceil 2 \cdot d !^{1 /(d-1)}\right\rceil$. We divide $B^{\prime}(A)$ into $s(d)^{d}$ congruent pieces by slicing parallel to each opposite pair of facets $s(d)$ times. Each piece has boundary measure $\left|\partial B^{\prime}(A)\right| / s(d)^{d-1}<\left|\partial B^{\prime}(A)\right| / 2^{d-1} d$ !. By the previous lemma, this is less than $|\partial A|$, so no piece can contain more than $k-1$ points. Thus, $B^{\prime}(A)$ contains at most $s(d)^{d}(k-1)=O(k)$ points. Since $C(A) \subset B^{\prime}(A), C(A)$ also contains $O(k)$ points. The points in $C(A)$ are the nearest orthogonal neighbors of $B_{d-2}(A)$.

Theorem 9.1. We can find the minimum boundary measure $k$-point subset of a set of $n$ points in $\mathbb{R}^{d}$, in time $O\left(n^{d}+2^{O(k)} n^{d-1}\right)$ and space $O(n)$.

We can generalize $L_{\infty}$ perimeter into higher dimensions as follows. We define the $L_{\infty}$ boundary measure of a set $A$ as the boundary measure of the smallest axis-parallel hyperrectangle enclosing $A$. Using techniques similar to those used to prove the previous theorem, we have the following result.

Theorem 9.2. We can find the minimum $L_{\infty}$ boundary measure $k$-point subset of a set of $n$ points in $\mathbb{R}^{d}$, in time $O\left(n^{d}+k^{2 d-1} n^{d-1}\right)$ and space $O(n)$.

\subsection{Volume}

While it is possible to derive a relatively efficient minimum volume algorithm using orthogonal neighbors, we can do better if we use vertical neighbors, as in our minimum-area algorithm. We say that a point $p$ is a vertical neighbor of a polytope $T$ if the line through $p$ parallel to the $d$ th coordinate axis intersects $T$.

Given a set $A$ in $\mathbb{R}^{d}$ and an arbitrary point $p_{0}^{v} \in A$, we define a series of vertical extremal points, extremal simplices, and bounding boxes, which we denote $p_{i}^{v}, S_{i}^{v}$, and $B_{i}^{\mathrm{v}}$, respectively. As before, we define $S_{0}^{\mathrm{v}}=B_{0}^{\mathrm{v}}=p_{0}^{\mathrm{v}}$. For all $1 \leq i \leq d$, $p_{i}^{\mathrm{v}}$ is the point in $A$ farthest along the $i$ th coordinate axis from aff $\left(S_{i-1}^{\mathrm{v}}\right) . S_{i}^{\mathrm{v}}=\operatorname{conv}\left(S_{i-1}^{\mathrm{v}}, p_{i}^{\mathrm{v}}\right)$. $B_{i}^{v}$ is the convex hull of two copies of $B_{i-1}^{v}$, displaced equal distances in opposite directions along the $i$ th coordinate axis, one containing $p_{i}^{v}$. For any set $A$, we have $S_{d}^{\mathrm{v}}(A) \subset A \subset B_{d}^{\mathrm{v}}(A)$. Clearly, Lemma 9.1 still holds if we consider vertical neighbors to $B_{r}^{\mathrm{v}}(A)$ instead of orthogonal neighbors to $B_{r}(A)$, and Lemma 9.2 also applies to vertical bounding boxes and extremal simplices.

Lemma 9.5. The minimum volume set $A$ is contained in the $O(k)$ nearest vertical neighbors of $B_{d-1}^{\mathrm{v}}(A)$. 
Proof. We divide $B_{d}^{v}(A)$ into $2^{d} d$ ! congruent convex pieces. By Lemma 9.2, each piece has the same volume as $S_{d}^{\mathrm{v}}(A)$. Since $|A| \geq\left|S_{d}^{\mathrm{v}}(A)\right|$, no piece can contain more than $k$ points. The points in $B_{d}^{\mathrm{v}}(A)$ are the nearest vertical neighbors of $B_{d-1}^{v}(A)$.

We now describe an efficient algorithm for finding nearest vertical neighbors to $(d-1)$-dimensional boxes. First consider the simpler problem of finding nearest neighbors to hyperplanes. As we did in our planar algorithm, we use geometric duality to transform the problem into finding, in an arrangement of hyperplanes, the $k$ closest hyperplanes above some query point. Vertical pointhyperplane distances in the dual space are the same as the corresponding vertical hyperplane-point distances in the primal space. Thus, we can solve this problem by vertical ray shooting in the dual space. We use the following result of Matoušek [28].

Lemma 9.6 [28]. We can preprocess a set of $n$ points in $\mathbb{R}^{d}$, in time and space $O\left(n^{d} / \log ^{d-1} n\right)$, so that the $k$ nearest neighbors to a query hyperplane can be found in time $O(k \log n)$.

We make use of a technique developed by Chazelle et al. [9] for answering simplex range queries. Given a data structure used to answer some arbitrary geometric query, they build on top of it another structure, called a partition tree, that limits the query to the points within an arbitrary half-space. The resulting data structure can be built in time $O\left(n^{d+\varepsilon}+P(n)\right)$, where $P(n)$ is the preprocessing time required for the original structure; and queries are answered in time $O(Q(n) \log n)$, where $Q(n)$ is the original query time. By building several levels of partition trees, it is possible to limit queries to the intersection of several half-spaces.

Lemma 9.7. We can preprocess a set of $n$ points in $\mathbb{R}^{d}$, in time and space $O\left(n^{d} / \log ^{d-1} n\right)$, so that the $k$ nearest vertical neighbors to a query $(d-1)$-dimensional box can be found in time $O\left(k \log ^{d+1} n\right)$.

Proof. It suffices to find vertical neighbors to simplices, since every box can be split into a constant number of simplices, and neighbors can be merged in time $O(k)$. We build a $d$-level partition tree, one level for each $(d-2)$-face of the query simplex, on top of Matoušek's vertical ray-shooting data structure. Since the hyperplanes bounding the query region are all vertical, we actually build the partition trees in $\mathbb{R}^{d-1}$, by ignoring the $d$ th coordinate of every point.

Theorem 9.3. We can find the minimum volume $k$-point subset of a set of $n$ points in $\mathbb{R}^{d}$, in time $O\left(k n^{d} \log ^{d+1} n+2^{O(k)} n^{d}\right)$ and space $O\left(n^{d-1+\varepsilon}\right)$. 


\section{Finding Minimal Convex Sets}

We wish to extend our results to the problem of finding minimal convex $k$-point sets, that is, sets of $k$ points which are the vertices of some convex polygon or polytope. Our previous results were based on the fact that, if some $k$ points are contained in a small convex body, we can find a $k$-point set with smaller perimeter (circumradius, diameter, convex hull volume, etc.) than that body. We cannot apply this technique directly, since $k$ points contained in some convex body obviously do not necessarily form a convex set. Hence we need a result of the form that, if enough points are given, some $k$-point subset will be convex. Such results are given by Ramsey theory [24]; indeed, the following was one of the seminal results in the development of both Ramsey theory and combinatorial geometry.

Lemma 10.1 [21]. Given a set of

$$
E S_{2}(k) \leq\left(\begin{array}{c}
2 k-4 \\
k-2
\end{array}\right)+1
$$

points in general position in the plane, some $k$ points form the vertices of $a$ convex polygon.

Lemma 10.2. Given a set of

$$
E S_{d}(k) \leq\left(\begin{array}{c}
2 k-4 \\
k-2
\end{array}\right)+1
$$

points in general position in $\mathbb{R}^{d}$, some $k$ points form the vertices of a convex polytope.

Proof. Project any set of $E S_{2}(k)$ points in $\mathbb{R}^{d}$ down to any plane. By the previous lemma, some $k$ points in the projection form a convex polygon. The preimage of those $k$ points forms a convex polytope in $\mathbb{R}^{d}$.

This gives us an upper bound of $E S_{d}(k)=O\left(4^{k}\right)$. Erdös and Szekeres also conjecture that $E S_{2}(k)=2^{k-2}+1$ and prove that this is a lower bound. Tightening the bounds on this function remains one of the outstanding open problems in combinatorial geometry [11]. We know of no bounds on $E S_{d}(k)$ other than those stated here, but it is clear that the function decreases with increasing $d$. Clearly, any reduction of the upper bound on $E S_{d}(k)$ would speed up our algorithms.

Using the previous lemma, we can generalize all of our results, both static and dynamic, to find minimum measure convex sets. The resulting time bounds have the same dependence on $n$ as the corresponding $k$-point set results, but with an exponential dependence on $k$.

For each of the measures we consider, if the minimum measure set is contained in the $m$ nearest neighbors of each of its points, then the minimum measure convex 
set is contained in the $O\left(m 4^{k} / k\right)$ nearest neighbors of each of its points. Our proof technique is identical to the one used for our earlier neighbor-counting lemmas. We describe a convex body, typically a hypercube, that contains the minimum measure set. We then divide the body into small pieces, such that if any piece contains $O\left(4^{k}\right)$ points, then it necessarily contains a $k$-point convex set with smaller measure than the original minimum measure set.

Theorem 10.1. Given a set of $n$ points in the plane, we can find the convex $k$-gon with minimum perimeter or $L_{\infty}$ perimeter, in time $O\left(n \log n+2^{6 k} n\right)$ and space $O\left(n \log n+4^{k} n+2^{4 k}\right)$. We can maintain the convex $k$-gon with minimum perimeter or $L_{\infty}$ perimeter as points are inserted, in time $O\left(2^{6 k} k+\log ^{2} n\right)$ per insertion and space $O\left(n \log n+2^{4 k}\right)$.

Proof. The minimum perimeter convex $k$-gon is contained in the $O\left(4^{k}\right)$ nearest neighbors to each of its points. We can use the dynamic programming algorithm of Eppstein et al. [20] to find minimum perimeter convex $k$-gons in time $O\left(\mathrm{kn}^{3}\right)$ and space $O\left(n^{2}\right)$. Using their algorithm as a subroutine, we achieve a static time bound of $O\left(n \log n+k\left(4^{k}\right)^{3} n / k\right)=O\left(n \log n+2^{6 k} n\right)$. The dynamic time bound follows directly from Lemma 6.1. Our algorithms work under any metric.

Theorem 10.2. Given a set of $n$ points in the plane, we can find the convex $k$-gon with minimum circumradius or $L_{\infty}$ diameter, in time $O\left(n \log n+2^{10 k} n / k\right)$ and space $O\left(n \log n+4^{k} n\right)$. We can maintain the convex $k$-gon with minimum circumradius or $L_{\infty}$ diameter as points are inserted, in time $O\left(2^{10 k}+\log ^{2} n\right)$ per insertion and space $O\left(n \log n+4^{k}\right)$.

Proof. The minimum circumradius convex $k$-point set is contained in the $O\left(4^{k}\right)$ nearest neighbors to each of its points. Edelsbrunner and Guibas [15] describe an algorithm that finds, given a set of $n$ points, the largest (cardinality) convex subset that includes a given leftmost point, in time $O\left(n^{2}\right)$ and space $O(n)$. For each point $p$ and each circumcircle containing it, rotate the points within the circle so that $p$ is leftmost, and find the largest convex subset containing $p$. Since each point is on $O\left(n^{2}\right)$ circumcircles, the resulting algorithm finds the minimum circumradius convex $k$-gon in time $O\left(n^{5}\right)$ and space $O(n)$. We use this algorithm as a subroutine.

Theorem 10.3. Given $a$ set of $n$ points in the plane, we can find the convex $k$-gon with minimum area, in time $O\left(n^{2} \log n+2^{6 k} n^{2}\right)$ and space $O\left(n \log n+2^{4 k}\right)$.

Proof. The minimum area convex $k$-point sets is contained in the $O\left(4^{k}\right)$ nearest vertical neighbors to the segment joining its leftmost and rightmost points. We can use the dynamic programming algorithm of Eppstein et al. [20] to find minimum area convex $k$-gons in time $O\left(k n^{3}\right)$ and space $O\left(n^{2}\right)$. As in the $k$-point set problem, we can reduce the time complexity by combining $j$-gons, $j<k$, from each side of each segment $p_{\mathrm{L}} p_{\mathrm{R}}$. As in the $k$-point set problem, all minimum $j$-gons 
Table 3. New results for finding minimum measure convex $k$-gons, given $n$ points in the plane. (Compare Table 1.)

\begin{tabular}{lll}
\hline \multicolumn{1}{c}{ Measure } & \multicolumn{1}{c}{ Static time bound } & Dynamic time bound \\
\hline Perimeter & $O\left(n \log n+2^{6 k} n\right)$ & $O\left(2^{6 k} k+\log ^{2} n\right)$ \\
$L_{\infty}$ perimeter & $O\left(n \log n+2^{6 k} n\right)$ & $O\left(2^{6 k} k+\log ^{2} n\right)$ \\
Circumradius & $O\left(n \log n+2^{10 k} n / k\right)$ & $O\left(2^{10 k}+\log ^{2} n\right)$ \\
$L_{\infty}$ diameter & $O\left(n \log n+2^{10 k} n / k\right)$ & $O\left(2^{10 k}+\log ^{2} n\right)$ \\
Diameter & $O\left(n \log n+2^{2 k^{2}+O(k)} n\right)$ & $O\left(2^{2 k^{2}+O(k)}+\log ^{2} n\right)$ \\
Variance & $O\left(n \log n+2^{2 k^{2}+k \lg k+O(k)} n\right)$ & $O\left(2^{2 k^{2}+k \lg k+O(k)}+\log ^{2} n\right)$ \\
\hline Area & $O\left(n^{2} \log n+2^{6 k} n^{2}\right)$ & \\
\hline
\end{tabular}

can be computed in time $O\left(\left(4^{k}\right)^{3}\right)=O\left(2^{6 k}\right)$, and the results can be combined in time $O(k)$. Therefore the total time is $O\left(n^{2} \log n+2^{6 k} n^{2}\right)$.

We are unable to generalize our planar diameter and variance algorithms, or any of our algorithms in higher dimensions, to find minimal convex sets. Consequently, we must use brute force within the neighbor sets, and our resulting time bounds are heavily exponential in $k$. Nevertheless, for sufficiently small $k$, our algorithms are faster than brute force. We summarize our planar results in Table 3 , and our higher-dimensional results in Table 4.

\section{Conclusions and Open Problems}

We have presented several algorithms for finding minimum measure $k$-point sets under a variety of measures, both in the plane and in higher dimensions. Our results are based on a common method. Given a set of points, we compute the nearest neighbors to each subset of $r$ points, where $r$ is a small constant determined by the relevant measure, and then search within each neighbor set using another algorithm. For most of the measures we have examined, $r=1$. For these measures,

Table 4. New results for finding minimum measure $k$-vertex convex polytopes, given $n$ points in $\mathbb{R}^{d}$, for all $d>2$. (Compare Table 2.)

\begin{tabular}{ll}
\hline \multicolumn{1}{c}{ Measure } & \multicolumn{1}{c}{ Time bound } \\
\hline Circumradius & $O\left(2^{2 k} n \log n+2^{2 k^{2}+O(k)} n\right)$ \\
Diameter & $O\left(2^{2 k} n \log n+2^{2 k^{2}+O(k)} n\right)$ \\
$L_{\infty}$ diameter & $O\left(2^{2 k} n \log n+2^{2 k^{2}+O(k)} n\right)$ \\
Variance & $O\left(4^{k} k^{(d-1) / 2} n \log n+2^{2 k^{2}+((d-1) / 2) k \lg k+O(k)} n\right)$ \\
\hline Boundary measure & $O\left(n^{d}+2^{2 k^{2}+O(k)} n^{d-1}\right)$ \\
$L_{\infty}$ boundary measure & $O\left(n^{d}+2^{2 k^{2}+O(k)} n^{d-1}\right)$ \\
Volume & $O\left(k n^{d} \log ^{d+1} n+2^{2 k^{2}+O(k)} n^{d}\right)$ \\
\hline
\end{tabular}


we can reduce the number of neighbor sets to search down to $O(n / k)$ by finding neighbors of neighbors. Our planar results were achieved through the use of a new algorithm that finds the $m$ nearest rectilinear neighbors of each of a given set of $n$ points, in time $O(n \log n+m n)$ on a random access machine. We have also presented versions of our algorithms which maintain minimum measure sets as points are inserted and versions which find, or dynamically maintain, minimum measure convex sets.

Eppstein et al. [20] also consider the problem of finding minimal empty convex polygons. Unfortunately, our methods do not suffice to solve this problem, except for a few special cases. In the plane every set of five points contains an empty convex quadrilateral, and every set of ten points contains an empty convex pentagon; our techniques can be applied to these cases. However, it is open whether there is a largest set with no empty convex hexagon (see, for example, [31]), and there are arbitrarily large point sets that contain no empty convex heptagons [26]. We know of no similar results in higher dimensions.

Our results suggest several open problems. None of our results is known to be optimal. Faster algorithms, or matching lower bounds, would be interesting. In particular, is it possible to find higher-dimensional $k$-point sets with minimum diameter, volume, or boundary measure without resorting to brute force? Dobkin et al. [14] and Eppstein et al. [20] present dynamic programming algorithms which can be used to solve a variety of minimum and maximum measure problems in the plane, but it seems highly unlikely that their approach can be adapted to higher-dimensional problems. Similarly, we have been unable to generalize our minimum-diameter algorithm, or the earlier algorithms of Aggarwal et al. [2], into higher dimensions.

Are there faster algorithms for finding nearest neighbors? An efficient technique for finding neighbors of $(d-2)$-flats might also lead to a faster mimimum-boundary-measure algorithm. Finally, is it possible to find rectilinear neighbors of points in higher dimensions in $o(m n \log n)$ time?

\section{Recent Results}

Dickerson et al. [13] describe an algorithm for finding $k$ nearest neighbors in the plane, under any metric, in time $O(n \log n+k n \log k)$ and space $O(n)$. Except for $L_{\infty}$ diameter, we can replace our neighbor algorithm with theirs in each of the algorithms in Section 5, without decreasing the time. The resulting algorithms all follow the algebraic decision-tree model of computation. In fact, we can improve the performance of our variance algorithm by substituting the new neighbor algorithm for Vaidya's. The new algorithm is faster than the one described by Aggarwal et al. [2] for all values of $k$.

Theorem 12.1. We can find the minimum variance $k$-point subset of a set of $n$ points in the plane, in time $O\left(n \log n+k^{3 / 2+\varepsilon} n\right)$ and space $O\left(k n+k^{5 / 2}\right)$. 
Very recently, Datta et al. [12] developed new algorithms for each of the problems we discuss in Section 5, except variance, using a search technique developed by Lenhof and Smid [27] that does not require the computation of nearest neighbors and follows the algebraic decision-tree model. In the plane their algorithms use the same time and less space than the solutions we present here; in higher dimensions they improve both both time and space bounds. They also present faster algorithms for maintaining the optimal set as points are inserted, and new algorithms for maintaining the optimal set as points are inserted and deleted, using techniques developed by Smid [33]. Their dynamic algorithms work efficiently in all dimensions.

\section{References}

1. P. K. Agarwal and J. Matoušek. Ray shooting and parametric search. In Proc. 24th ACM Symp. Theory Comput., pp. 517-526, 1992.

2. A. Aggarwal, H. Imai, N. Katoh, and S. Suri. Finding $k$ points with minimum diameter and related problems. J. Algorithms, 12:38-56, 1991.

3. M. Ajtai, J. Komlós, and E. Szemerédi. Sorting in $c \log n$ parallel steps. Combinatorica, 3:1-19, 1983.

4. J. L. Bentley and J. B. Saxe. Decomposable searching problems, I: Static-to-dynamic transformation. J. Algorithms, 1:301-358, 1980 .

5. B. Chazelle. An optimal convex hull algorithm and new results on cuttings. In Proc. 32nd IEEE Symp. Found. Comput. Sci., pp. 29-38, 1991.

6. B. Chazelle and H. Edelsbrunner. An improved algorithm for constructing $k$ th-order Voronoi diagrams. IEEE Trans. Comput., 36:1349-1354, 1987.

7. B. Chazelle, L. J. Guibas, and D. T. Lee. The power of geometric duality. BIT, 25:76-90, 1985.

8. B. M. Chazelle and D. T. Lee. On a circle placement problem. Computing, 36:1-16, 1986.

9. B. Chazelle, M. Sharir, and E. Welzl. Quasi-optimal upper bounds for simplex range searching and new zone theorems. In Proc. 6th ACM Symp. Comput. Geom., pp. 23-33, 1990.

10. R. Cole. Slowing down sorting networks to obtain faster sorting algorithms. J. Assoc. Comput. Mach., 34:200-208, 1987.

11. H. P. Croft, K. J. Falconer, and R. K. Guy. Unsolved Problems in Geometry. Springer-Verlag, New York, 1990.

12. A. Datta, H.-P. Lenhof, C. Schwarz, and M. Smid. Static and dynamic algorithms for $k$-point clustering problems. In Proc. 3rd Workshop Algorithms Data Struct., pp. 265-276. Lecture Notes in Computer Science, vol. 709. Springer-Verlag, New York, 1993.

13. M. T. Dickerson, R. L. Drysdale, and J. R. Sack. Simple algorithm for enumerating interpoint distances and finding $k$ nearest neighbors. Internat. J. Comput. Geom. Appl., 2:221-239, 1993.

14. D. P. Dobkin, R. L. Drysdale, and L. J. Guibas. Finding smallest polygons. In F. P. Preparata, ed., Computational Geometry, pp. 181-214. Advances in Computing Research, vol. 1. JAI Press, Greenwich, CT, 1983.

15. H. Edelsbrunner and L. J. Guibas. Topologically sweeping an arrangement. J. Comput. System. Sci., 38:165-194, 1989.

16. H. Edelsbrunner, J. O'Rourke, and R. Seidel. Constructing arrangements of lines and hyperplanes with applications. SIAM J. Comput., 15:341-363, 1986.

17. D. Eppstein. New algorithms for minimum area $k$-gons. In Proc. 3rd ACM-SIAM Symp. Discrete Algorithms, pp. 83-88, 1992.

18. D. Eppstein. Persistence, offline algorithms, and space compaction. Technical Report 91-54, Dept. Information and Computer Science, University of California, Irvine, 1991.

19. D. Eppstein and J. Erickson. Iterated nearest neighbors and finding minimal polytopes. In Proc. 4th ACM-SIAM Symp. Discrete Algorithms, pp. 64-73, 1993. 
20. D. Eppstein, M. Overmars, G. Rote, and G. Woeginer. Finding minimum area $k$-gons. Discrete Comput. Geom., 7:45-58, 1992.

21. P. Erdős and G. Szekeres. A combinatorial problem in geometry. Compositio Math., 2:463-470, 1935.

22. G. N. Frederickson and D. B. Johnson. The complexity of selection and raking in $X+Y$ and matrices with sorted rows and columns. J. Comput. System Sci. 24:197-208, 1982.

23. F. W. Fredman and D. E. Willard. Trans-dichotomous algorithms for minimum spanning trees and shortest paths. In Proc. 31st IEEE Symp. Found. Comput. Sci., pp. 719-725, 1990.

24. R. L. Graham, B. L. Rothschild, and J. H. Spencer. Ramsey Theory, 2nd edn. Wiley, New York, 1990.

25. J. Hershberger and S. Suri. Finding tailored paritions. In Proc. 5th ACM Symp. Comput. Geom., pp. 255-265, 1989.

26. J. D. Horton. Sets with no empty convex 7-gons. Canad. Math. Bull, 26:482-484, 1983.

27. H.-P. Lenhof and M. Smid. Enumerating the $k$ closest pairs optimally. In Proc. 33rd IEEE Symp. Found. Comput. Sci., pp. 380-386, 1992.

28. J. Matoušek. On vertical ray-shooting in arrangements. Comput. Geom. Theory. Appl., 2:279-285, 1993.

29. N. Megiddo. Applying parallel computation algorithms in the design of serial algorithms. J. Assoc. Comput. Mach., 30:852-865, 1983.

30. K. Mulmuley. Output sensitive construction of levels and Voronoi diagrams in $R^{d}$ of order 1 to k. In Proc. 22nid ACM Symp. Theory Comput., pp. 322-330, 1990.

31. M. H. Overmars, B. Scholten, and I. Vincent. Sets without empty convex 6-gons. Bull. EATCS, 37:160, 1989.

32. M. H. Overmars and C.-K. Yap. New upper bounds in Klee's measure problem. SIAM J. Comput., 20:1034-1045, 1991.

33. M. Smid. Maintaining the minimal distance of a point set in polylogarithmic time. Discrete Comput. Geom., 7:415-431, 1992.

34. P. M. Vaidya. An $O(n \log n)$ algorithm for the all-nearest-neighbors problem. Discrete Comput. Geom., 4:101-115, 1989.

Received July 9, 1992, and in revised form July 28, 1993. 